Research Article

\title{
Binomial tribonacci sums
}

\author{
Kunle Adegoke ${ }^{1, *}$, Robert Frontczak ${ }^{2}$, Taras Goy ${ }^{3}$
}

${ }^{1}$ Department of Physics and Engineering Physics, Obafemi Awolowo University, Ile-Ife, Nigeria

${ }^{2}$ Landesbank Baden-Württemberg, Stuttgart, Germany

${ }^{3}$ Faculty of Mathematics and Computer Science, Vasyl Stefanyk Precarpathian National University, Ivano-Frankivsk, Ukraine

(Received: 20 August 2021. Received in revised form: 28 September 2021. Accepted: 6 October 2021. Published online: 13 October 2021.)

(C) 2021 the authors. This is an open access article under the CC BY (International 4.0) license (www.creativecommons.org/licenses/by/4.0/).

\section{Abstract}

We derive expressions for several binomials sums involving a generalized tribonacci sequence. We also study double binomial sums involving this sequence. Several explicit examples involving tribonacci and tribonacci-Lucas numbers are stated to highlight the results.

Keywords: generalized tribonacci sequence; tribonacci number; tribonacci-Lucas number; binomial transform.

2020 Mathematics Subject Classification: 11B37, 11B39.

\section{Introduction}

There is a dearth of tribonacci summation identities including binomial coefficients. Our goal in this paper is to derive several new binomial tribonacci sums such as

$$
\begin{gathered}
\sum_{k=0}^{n}\left(\begin{array}{l}
n \\
k
\end{array}\right) G_{4 k+s}=2^{n} G_{3 n+s}, \quad \sum_{k=1}^{n}\left(\begin{array}{l}
n \\
k
\end{array}\right) \frac{G_{4 k+s}}{k}=\sum_{m=1}^{n} \frac{2^{m} G_{3 m+s}-G_{s}}{m}, \\
\sum_{k=0}^{\lfloor 3 n / 2\rfloor}\left(\begin{array}{c}
3 n \\
2 k
\end{array}\right) G_{2 k+s}=2^{n-1}\left(G_{4 n+s}+(-1)^{n} G_{s-2 n}\right), \quad \sum_{k=0}^{n}(-1)^{n-k}\left(\begin{array}{c}
n+k \\
2 k
\end{array}\right) \frac{4^{k}}{n+k} G_{4 n+2 k+s}=\frac{G_{8 n+s}+G_{s}}{2 n},
\end{gathered}
$$

and double binomial tribonacci summation identities such as

$$
\sum_{k=0}^{n} \sum_{p=0}^{k}(-1)^{k+p}\left(\begin{array}{l}
n \\
k
\end{array}\right)\left(\begin{array}{l}
k \\
p
\end{array}\right) G_{5 k+p+s}=3^{n} G_{3 n+s}, \quad \sum_{k=0}^{n} \sum_{p=0}^{k}\left(\begin{array}{l}
n \\
k
\end{array}\right)\left(\begin{array}{l}
k \\
p
\end{array}\right) \frac{G_{k+5 p+s}}{3^{k}}=\left(\frac{7}{3}\right)^{n} G_{3 n+s} .
$$

In the above identities, $n$ denotes a non-negative integer, $s$ and $p$ are arbitrary integers and $G_{n}$ is a generalized tribonacci number.

The generalized tribonacci sequence $G_{n}=G_{n}\left(c_{0}, c_{1}, c_{2}\right), n \geq 0$, is defined recursively by

$$
G_{n}=G_{n-1}+G_{n-2}+G_{n-3}, \quad n \geq 3,
$$

with initial values $G_{0}=c_{0}, G_{1}=c_{1}, G_{2}=c_{2}$ not all being zero. Extension of the definition of $G_{n}$ to negative subscripts is provided by writing the recurrence relation as

$$
G_{-n}=G_{-(n-3)}-G_{-(n-2)}-G_{-(n-1)}
$$

so that $G_{n}$ is defined for all integers $n$.

The most prominent representatives of $G_{n}$ and widely studied in the literature are $G_{n}(0,1,1)=T_{n}$ the sequence of tribonacci numbers and $G_{n}(3,1,3)=K_{n}$ the sequence of tribonacci-Lucas numbers (sequences A000073 and A001644 in [19], respectively).

The first few tribonacci numbers and tribonacci-Lucas numbers with positive and negative subscripts are given in Table 1.

${ }^{*}$ Corresponding author (adegoke00@gmail.com). 


\begin{tabular}{|c||c|c|c|c|c|c|c|c|c|c|c|c|c|c|c|}
\hline$n$ & 0 & 1 & 2 & 3 & 4 & 5 & 6 & 7 & 8 & 9 & 10 & 11 & 12 & 13 & 14 \\
\hline \hline$T_{n}$ & 0 & 1 & 1 & 2 & 4 & 7 & 13 & 24 & 44 & 81 & 149 & 274 & 504 & 927 & 1705 \\
\hline$T_{-n}$ & 0 & 0 & 1 & -1 & 0 & 2 & -3 & 1 & 4 & -8 & 5 & 7 & -20 & 18 & 9 \\
\hline$K_{n}$ & 3 & 1 & 3 & 7 & 11 & 21 & 39 & 71 & 131 & 241 & 443 & 815 & 1499 & 2757 & 5071 \\
\hline$K_{-n}$ & 3 & -1 & -1 & 5 & -5 & -1 & 11 & -15 & 3 & 23 & -41 & 21 & 43 & -105 & 83 \\
\hline
\end{tabular}

Table 1: Tribonacci and tribonacci-Lucas numbers.

Properties of (generalized) tribonacci sequences were investigated in the recent articles [1-4, 7, 8, 10, 12-18, 20, 21], among others. For instance, Janjić [16] found the remarkable combinatorial identity

$$
T_{n}=1+\sum_{k=1}^{n-1} \sum_{i=0}^{k} \sum_{j=i}^{n-k}\left(\begin{array}{l}
k \\
i
\end{array}\right)\left(\begin{array}{l}
j-1 \\
i-1
\end{array}\right)\left(\begin{array}{c}
j \\
n-k-2 j
\end{array}\right) .
$$

A generalized tribonacci number $G_{n}\left(c_{0}, c_{1}, c_{2}\right)$ is given by the Binet formula

$$
G_{n}\left(c_{0}, c_{1}, c_{2}\right)=A \alpha^{n}+B \beta^{n}+C \gamma^{n},
$$

where $\alpha, \beta$ and $\gamma$ are the distinct roots of the equation $x^{3}-x^{2}-x-1=0$. The coefficients $A, B$ and $C$ depend on the initial values and are determined by the system

$$
\left\{\begin{array}{l}
A+B+C=c_{0}, \\
A \alpha+B \beta+C \gamma=c_{1}, \\
A \alpha^{2}+B \beta^{2}+C \gamma^{2}=c_{2} .
\end{array}\right.
$$

The Binet formulas for $T_{n}$ and $K_{n}$ are

$$
T_{n}=\frac{\alpha^{n+1}}{(\alpha-\beta)(\alpha-\gamma)}+\frac{\beta^{n+1}}{(\beta-\alpha)(\beta-\gamma)}+\frac{\gamma^{n+1}}{(\gamma-\alpha)(\gamma-\beta)}
$$

and

$$
K_{n}=\alpha^{n}+\beta^{n}+\gamma^{n}
$$

where

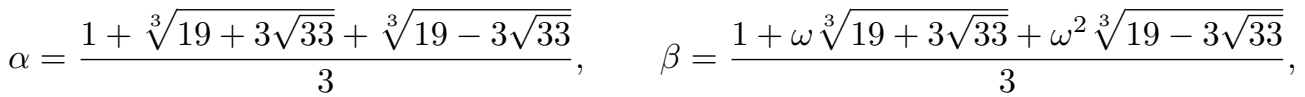

$$
\begin{aligned}
& \gamma=\frac{1+\omega^{2} \sqrt[3]{19+3 \sqrt{33}}+\omega \sqrt[3]{19-3 \sqrt{33}}}{3}
\end{aligned}
$$

and $\omega=\frac{-1+i \sqrt{3}}{2}$ is a primitive cube root of unity.

Tribonacci and tribonacci-Lucas numbers with negative indices can be accessed directly, using the following result.

Lemma 1.1. For integer $n$,

$$
\begin{gathered}
T_{-n}=T_{n-1}^{2}-T_{n-2} T_{n}, \\
K_{-n}=\frac{K_{n}^{2}-K_{2 n}}{2} .
\end{gathered}
$$

For a proof of (2), see, for example, [8, Theorem 2.2]. The proof of (3) one can find in [6, Formula (9)].

In this article, we study binomial and double binomial sums with terms being a generalized tribonacci sequence. We derive closed forms for several such sums. We also prove a general binomial identity characterizing $G_{a n+b}$ for $a \geq 1$ and $b$ an arbitrary integer.

\section{Some auxiliary results}

In this section we present some results that we will use in the sequel.

Lemma 2.1. Let $\phi \in\{\alpha, \beta, \gamma\}$. Then, for all $n \geq 0$, we have

$$
\phi^{n+1}=\phi^{2} T_{n}+\phi\left(T_{n-1}+T_{n-2}\right)+T_{n-1} .
$$

For a proof of (4), see [7, Formula (6)]. 
Lemma 2.2. We have

$$
\begin{aligned}
(\alpha-1)^{3} & =2 \alpha^{-2}, \\
(\alpha+1)^{3} & =2 \alpha^{4}, \\
\left(\alpha^{2}+1\right)^{3} & =4 \alpha^{5}, \\
\left(\alpha^{3}-1\right)^{3} & =2 \alpha^{7}, \\
\alpha^{4}+1 & =2 \alpha^{3},
\end{aligned}
$$

with identical relations for $\beta$ and $\gamma$.

Proof. Since

$$
1+\alpha+\alpha^{2}=\alpha^{3},
$$

we have

$$
\frac{\alpha^{2}+1}{\alpha^{2}-1}=\alpha
$$

and

$$
\frac{\alpha+1}{\alpha-1}=\alpha^{2} .
$$

Addition of (11) and (12) gives

$$
(\alpha+1)^{2}(\alpha-1)=2 \alpha^{2},
$$

while their subtraction produces

$$
(\alpha-1)^{2}(\alpha+1)=2 .
$$

Eliminating $\alpha+1$ between (13) and (14) gives identity (5), while the elimination of $\alpha-1$ yields (6).

Cubing identity $\alpha^{2}+1=\frac{2 \alpha}{\alpha-1}$ and making use of (5) gives (7). Subtracting (10) from $\alpha+\alpha^{2}+\alpha^{3}=\alpha^{4}$ produces identity (8). Identity (9) follows from $\alpha^{4}+1=\alpha^{4}+\alpha^{3}+\alpha^{2}+\alpha=\left(\alpha^{2}+1\right)(\alpha+1)$ with the help of (6) and (7).

Lemma 2.3. Let $a, b, c$ and $d$ be rational numbers and $\lambda$ an irrational number. Then

$$
a+\lambda b=c+\lambda d \quad \Longleftrightarrow \quad a=c, \quad b=d .
$$

\section{Identities from the binomial theorem and binomial transform}

The next lemma will be the key ingredient to derive many results in this paper. For a proof and some applications to Horadam numbers, see [11].

Lemma 3.1. Let $n$ and $j$ be integers with $0 \leq j \leq n$. Then, for each $x, y \in \mathbb{C}$, we have

$$
\sum_{k=j}^{n}( \pm 1)^{k-j}\left(\begin{array}{l}
k \\
j
\end{array}\right)\left(\begin{array}{l}
n \\
k
\end{array}\right) y^{k} x^{n-k}=\left(\begin{array}{l}
n \\
j
\end{array}\right) y^{j}(x \pm y)^{n-j}
$$

We also mention the standard fact about sequences and their binomial transforms [5]: Let $\left(a_{n}\right)_{n \geq 0}$ be a sequence of numbers and $\left(b_{n}\right)_{n \geq 0}$ be its binomial transform. Then we have the following relations:

$$
b_{n}=\sum_{k=0}^{n}\left(\begin{array}{l}
n \\
k
\end{array}\right) a_{k} \quad \Longleftrightarrow \quad a_{n}=\sum_{k=0}^{n}\left(\begin{array}{l}
n \\
k
\end{array}\right)(-1)^{n-k} b_{k} .
$$

Furthermore, if $a_{0}=0$ (so that $b_{0}=0$ too) the binomial pair exhibits the following properties:

$$
\sum_{k=1}^{n}\left(\begin{array}{l}
n \\
k
\end{array}\right) \frac{a_{k}}{k}=\sum_{m=1}^{n} \frac{b_{m}}{m}
$$

and

$$
\sum_{k=1}^{n}\left(\begin{array}{l}
n \\
k
\end{array}\right) \frac{a_{k}}{k+1}=\frac{1}{n+1} \sum_{m=1}^{n} b_{m} .
$$

Theorem 3.1. Let $j$ and $s$ be integers such that $s$ is arbitrary and $j \geq 0$. Then

$$
\sum_{k=j}^{n}\left(\begin{array}{l}
k \\
j
\end{array}\right)\left(\begin{array}{l}
n \\
k
\end{array}\right) G_{4 k+s}=\left(\begin{array}{l}
n \\
j
\end{array}\right) 2^{n-j} G_{3 n+j+s}
$$


Proof. Use identity (9) in Lemma 3.1 with $x=1$ and $y=\alpha^{4}$, taking note of Lemma 2.3.

Corollary 3.1. For $n$ a non-negative integer and s any integer,

$$
\begin{gathered}
\sum_{k=0}^{n}\left(\begin{array}{l}
n \\
k
\end{array}\right) G_{4 k+s}=2^{n} G_{3 n+s}, \\
\sum_{k=0}^{n}\left(\begin{array}{l}
n \\
k
\end{array}\right)(-2)^{k} G_{3 k+s}=(-1)^{n} G_{4 n+s}, \\
\sum_{k=1}^{n}\left(\begin{array}{l}
n \\
k
\end{array}\right) \frac{G_{4 k+s}}{k}=\sum_{m=1}^{n} \frac{2^{m} G_{3 m+s}-G_{s}}{m}
\end{gathered}
$$

and

$$
\sum_{k=1}^{n}\left(\begin{array}{l}
n \\
k
\end{array}\right) \frac{G_{4 k+s}}{k+1}=\frac{1}{n+1}\left(\sum_{m=1}^{n} 2^{m} G_{3 m+s}-n G_{s}\right) .
$$

Proof. To obtain (19) set $j=0$ in (18). Identities (20), (21) and (22) follow form (15), (16) and (17), respectively.

From (19) and (20) we immediately obtain the following binomial tribonacci and tribonacci-Lucas relations.

Corollary 3.2. For $n \geq 0$,

$$
\begin{array}{cc}
\sum_{k=0}^{n}\left(\begin{array}{l}
n \\
k
\end{array}\right) T_{4 k}=2^{n} T_{3 n}, & \sum_{k=0}^{n}\left(\begin{array}{l}
n \\
k
\end{array}\right) K_{4 k}=2^{n} K_{3 n}, \\
\sum_{k=0}^{n}\left(\begin{array}{l}
n \\
k
\end{array}\right) T_{4 k-3 n+1}=2^{n}, & \sum_{k=0}^{n}\left(\begin{array}{l}
n \\
k
\end{array}\right) K_{4 k-3 n+1}=2^{n}, \\
\sum_{k=0}^{n}\left(\begin{array}{l}
n \\
k
\end{array}\right) T_{4 k-3 n}=0, \quad \sum_{k=0}^{n}\left(\begin{array}{l}
n \\
k
\end{array}\right) K_{4 k-3 n}=3 \cdot 2^{n} .
\end{array}
$$

Theorem 3.2. For non-negative integer $n$, any integer s, we have

$$
\sum_{k=0}^{3 n} \delta^{k}\left(\begin{array}{c}
3 n \\
k
\end{array}\right) G_{p k+s}=\delta^{n} 2^{q n} G_{r n+s}
$$

where the values of $\delta, p, q$ and $r$ as given in each column in Table 2.

\begin{tabular}{|c||c|c|c|c|}
\hline$\delta$ & -1 & 1 & 1 & -1 \\
\hline$p$ & 1 & 1 & 2 & 3 \\
\hline$q$ & 1 & 1 & 2 & 1 \\
\hline$r$ & -2 & 4 & 5 & 7 \\
\hline
\end{tabular}

Table 2: Values of $\delta, p, q$ and $r$ from Theorem 3.2.

Proof. Each of the identities (5)-(8) can be written as $\left(\alpha^{p}+\delta\right)^{3}=2^{q} \alpha^{r}$, where the values of $\delta, p, q$ and $r$ in each case are as given in each column in Table 2 . The identity of the theorem then follows from the binomial theorem and Lemma 2.3.

Lemma 3.2. For non-negative integer $n$ and real or complex $z$,

$$
\begin{gathered}
2 \sum_{k=0}^{\lfloor 3 n / 2\rfloor}\left(\begin{array}{l}
3 n \\
2 k
\end{array}\right) z^{2 k}=(1+z)^{3 n}+(1-z)^{3 n}, \\
2 \sum_{k=1}^{\lceil 3 n / 2\rceil}\left(\begin{array}{c}
3 n \\
2 k-1
\end{array}\right) z^{2 k-1}=(1+z)^{3 n}-(1-z)^{3 n} .
\end{gathered}
$$

Theorem 3.3. For non-negative integer $n$ and any integer $s$,

$$
\begin{gathered}
\sum_{k=0}^{\lfloor 3 n / 2\rfloor}\left(\begin{array}{c}
3 n \\
2 k
\end{array}\right) G_{2 k+s}=2^{n-1}\left(G_{4 n+s}+(-1)^{n} G_{s-2 n}\right), \\
\sum_{k=1}^{\lceil 3 n / 2\rceil}\left(\begin{array}{c}
3 n \\
2 k-1
\end{array}\right) G_{2 k+s-1}=2^{n-1}\left(G_{4 n+s}-(-1)^{n} G_{s-2 n}\right) .
\end{gathered}
$$


Proof. Set $z=\alpha$ in Lemma 3.2, make use of identities (5) and (6), noting Lemma 2.3 with $\lambda=\alpha$.

Setting $s=0$ in Theorem 3.3, we immediately obtain the following.

Corollary 3.3. For non-negative integer n,

$$
\begin{gathered}
\sum_{k=0}^{\lfloor 3 n / 2\rfloor}\left(\begin{array}{c}
3 n \\
2 k
\end{array}\right) G_{2 k}=2^{n-1}\left(G_{4 n}+(-1)^{n} G_{-2 n}\right), \\
\sum_{k=1}^{\lceil 3 n / 2\rceil}\left(\begin{array}{c}
3 n \\
2 k-1
\end{array}\right) G_{2 k-1}=2^{n-1}\left(G_{4 n}-(-1)^{n} G_{-2 n}\right) .
\end{gathered}
$$

As special cases of formulas above we have:

$$
\begin{gathered}
\sum_{k=0}^{\lfloor 3 n / 2\rfloor}\left(\begin{array}{c}
3 n \\
2 k
\end{array}\right) T_{2 k}=2^{n-1}\left(T_{4 n}+(-1)^{n}\left(T_{2 n-1}^{2}-T_{2 n-2} T_{2 n}\right)\right), \\
\sum_{k=1}^{\lceil 3 n / 2\rceil}\left(\begin{array}{c}
3 n \\
2 k-1
\end{array}\right) T_{2 k-1}=2^{n-1}\left(T_{4 n}-(-1)^{n}\left(T_{2 n-1}^{2}-T_{2 n-2} T_{2 n}\right)\right)
\end{gathered}
$$

and

$$
\begin{gathered}
\sum_{k=0}^{\lfloor 3 n / 2\rfloor}\left(\begin{array}{c}
3 n \\
2 k
\end{array}\right) K_{2 k}=2^{n-2}\left(2 K_{4 n}+(-1)^{n}\left(K_{2 n}^{2}-K_{4 n}\right)\right), \\
\sum_{k=1}^{\lfloor 3 n / 2\rfloor}\left(\begin{array}{c}
3 n \\
2 k-1
\end{array}\right) K_{2 k-1}=2^{n-2}\left(2 K_{4 n}-(-1)^{n}\left(K_{2 n}^{2}-K_{4 n}\right)\right) .
\end{gathered}
$$

Theorem 3.4. For non-negative integer $n$ and any integer $s$,

$$
\begin{gathered}
\sum_{k=0}^{\lfloor 3 n / 2\rfloor}\left(\begin{array}{c}
3 n \\
2 k
\end{array}\right) G_{4 k+s}=2^{2 n-1}\left(G_{5 n+s}+(-1)^{n} G_{2 n+s}\right), \\
\sum_{k=1}^{\lceil 3 n / 2\rceil}\left(\begin{array}{c}
3 n \\
2 k-1
\end{array}\right) G_{4 k+s-2}=2^{2 n-1}\left(G_{5 n+s}-(-1)^{n} G_{2 n+s}\right) .
\end{gathered}
$$

Proof. Combining (5) with (6) yields

$$
\left(\alpha^{2}-1\right)^{3}=4 \alpha^{2} .
$$

Now set $z=\alpha^{2}$ in Lemma 3.2 and make use of identities (7) and (23), noting Lemma 2.3 with $\lambda=\alpha$.

Theorem 3.5. For non-negative integer $n$ and any integer $s$,

$$
\begin{gathered}
\sum_{k=0}^{\lfloor 3 n / 2\rfloor}\left(\begin{array}{c}
3 n \\
2 k
\end{array}\right) G_{8 k+s}=2^{3 n-1}\left(G_{9 n+s}+(-2)^{n} G_{7 n+s}\right), \\
\sum_{k=1}^{\lceil 3 n / 2\rceil}\left(\begin{array}{c}
3 n \\
2 k-1
\end{array}\right) G_{8 k+s-4}=2^{3 n-1}\left(G_{9 n+s}-(-2)^{n} G_{7 n+s}\right) .
\end{gathered}
$$

Proof. Combining (7) and (23) we have

$$
\left(\alpha^{4}-1\right)^{3}=16 \alpha^{7} .
$$

Set $z=\alpha^{4}$ in Lemma 3.2 and make use of identities (9) and (24), noting Lemma 2.3 with $\lambda=\alpha$. 


\section{Identities from the Waring formulas}

Our next result provides two combinatorial identities for generalized tribonacci numbers involving binomial coefficients.

Lemma 4.1. The following identities hold for $n \geq 0$ and real or complex $x$ and $y$ :

$$
\sum_{k=0}^{\lfloor n / 2\rfloor}(-1)^{k}\left(\begin{array}{c}
n-k \\
k
\end{array}\right)(x y)^{k}(x+y)^{n-2 k}=\frac{x^{n+1}-y^{n+1}}{x-y}
$$

and

$$
\sum_{k=0}^{\lfloor n / 2\rfloor}(-1)^{k}\left(\begin{array}{c}
n-k \\
k
\end{array}\right) \frac{n}{n-k}(x y)^{k}(x+y)^{n-2 k}=x^{n}+y^{n} .
$$

Formulas (25) and (26) are well-known in combinatorics and called Waring (sometimes Girard-Waring) formulas. The proof of these formulas can be found, for example, in [9].

Theorem 4.1. Let $n$ be a non-negative integer and s any integer. Then

$$
\sum_{k=0}^{\lfloor n / 2\rfloor}\left(-\frac{1}{4}\right)^{k}\left(\begin{array}{c}
n-k \\
k
\end{array}\right)\left(G_{3 n-2 k+s+4}-G_{3 n-2 k+s}\right)=\frac{G_{4 n+s+4}-G_{s}}{2^{n}}
$$

and

$$
\sum_{k=0}^{\lfloor n / 2\rfloor}\left(-\frac{1}{4}\right)^{k}\left(\begin{array}{c}
n-k \\
k
\end{array}\right) \frac{G_{3 n-2 k+s}}{n-k}=\frac{G_{4 n+s}+G_{s}}{2^{n} n} .
$$

Proof. Set $(x, y)=\left(1, \alpha^{4}\right)$ in (25) and (26), respectively, Lemma 4.1 and use identity (8) and Lemma 2.3.

Corollary 4.1. For $n \geq 0$,

In particular,

$$
\begin{gathered}
\sum_{k=0}^{\lfloor n / 2\rfloor}\left(-\frac{1}{4}\right)^{k}\left(\begin{array}{c}
n-k \\
k
\end{array}\right)\left(G_{n-2 k+4}-G_{n-2 k}\right)=\frac{G_{2 n+4}-G_{-2 n}}{2^{n}}, \\
\sum_{k=0}^{\lfloor n / 2\rfloor}\left(-\frac{1}{4}\right)^{k}\left(\begin{array}{c}
n-k \\
k
\end{array}\right) \frac{G_{n-2 k}}{n-k}=\frac{G_{2 n}+G_{-2 n}}{n 2^{n}} .
\end{gathered}
$$

$$
\begin{gathered}
\sum_{k=0}^{\lfloor n / 2\rfloor}\left(-\frac{1}{4}\right)^{k}\left(\begin{array}{c}
n-k \\
k
\end{array}\right)\left(T_{n-2 k+4}-T_{n-2 k}\right)=\frac{T_{2 n+4}-T_{2 n-1}^{2}+T_{2 n-2} T_{2 n}}{2^{n}}, \\
\sum_{k=0}^{\lfloor n / 2\rfloor}\left(-\frac{1}{4}\right)^{k}\left(\begin{array}{c}
n-k \\
k
\end{array}\right)\left(K_{n-2 k+4}-K_{n-2 k}\right)=\frac{2 K_{2 n+4}-K_{2 n}^{2}+K_{4 n}}{2^{n+1}}
\end{gathered}
$$

and

$$
\begin{gathered}
\sum_{k=0}^{\lfloor n / 2\rfloor}\left(-\frac{1}{4}\right)^{k}\left(\begin{array}{c}
n-k \\
k
\end{array}\right) \frac{T_{n-2 k}}{n-k}=\frac{T_{2 n-1}^{2}+T_{2 n}\left(1-T_{2 n-2}\right)}{n 2^{n}}, \\
\sum_{k=0}^{\lfloor n / 2\rfloor}\left(-\frac{1}{4}\right)^{k}\left(\begin{array}{c}
n-k \\
k
\end{array}\right) \frac{K_{n-2 k}}{n-k}=\frac{K_{2 n}^{2}+2 K_{2 n}-K_{4 n}}{n 2^{n+1}} .
\end{gathered}
$$

\section{Double binomial tribonacci sums}

Theorem 5.1. Let $n, j$ and $s$ be integers with s arbitrary and $j \geq 0$. Then,

$$
\sum_{k=j}^{n} \sum_{p=0}^{k}(-1)^{k-p}\left(\begin{array}{l}
k \\
j
\end{array}\right)\left(\begin{array}{l}
n \\
k
\end{array}\right)\left(\begin{array}{l}
k \\
p
\end{array}\right) G_{5 k+p+s}=3^{n-j}\left(\begin{array}{l}
n \\
j
\end{array}\right) \sum_{p=0}^{j}(-1)^{j-p}\left(\begin{array}{l}
j \\
p
\end{array}\right) G_{3 n+2 j+p+s} .
$$

Proof. The identity can be derived from Lemma 3.1 using $3 \phi^{3}=\phi^{6}-\phi^{5}+1$.

Corollary 5.1. Let $n$ and $s$ be integers. Then,

$$
\begin{gathered}
\sum_{k=0}^{n} \sum_{p=0}^{k}(-1)^{k-p}\left(\begin{array}{l}
n \\
k
\end{array}\right)\left(\begin{array}{l}
k \\
p
\end{array}\right) G_{5 k+p+s}=3^{n} G_{3 n+s}, \\
\sum_{k=1}^{n} \sum_{p=0}^{k}(-1)^{k-p}\left(\begin{array}{l}
n \\
k
\end{array}\right)\left(\begin{array}{l}
k \\
p
\end{array}\right) k G_{5 k+p+s}=n 3^{n-1}\left(G_{3 n+s}+G_{3 n+s+1}\right) .
\end{gathered}
$$


Proof. Set $j=0$ and $j=1$ in (27), respectively.

Theorem 5.2. Let $j$ and $s$ be integers with sarbitrary and $j \geq 0$. Then

$$
\begin{gathered}
\sum_{k=j}^{n} \sum_{p=0}^{k}\left(\begin{array}{l}
k \\
j
\end{array}\right)\left(\begin{array}{l}
n \\
k
\end{array}\right)\left(\begin{array}{l}
k \\
p
\end{array}\right) \frac{G_{k+4 p+s}}{2^{k}}=2^{2 n-j}\left(\begin{array}{l}
n \\
j
\end{array}\right) \sum_{m=0}^{j}\left(\begin{array}{c}
j \\
m
\end{array}\right) G_{3 n-2 j+4 m+s}, \\
\sum_{k=j}^{n} \sum_{p=0}^{k}\left(\begin{array}{l}
k \\
j
\end{array}\right)\left(\begin{array}{l}
n \\
k
\end{array}\right)\left(\begin{array}{l}
k \\
p
\end{array}\right) \frac{G_{k+5 p+s}}{3^{k}}=\frac{7^{n-j}}{3^{n}}\left(\begin{array}{c}
n \\
j
\end{array}\right) \sum_{m=0}^{j}\left(\begin{array}{c}
j \\
m
\end{array}\right) G_{3 n-2 j+5 m+s} .
\end{gathered}
$$

Proof. Use Lemma 3.1 in conjunction with $4 \phi^{3}=\phi^{5}+\phi+2$ and $7 \phi^{3}=\phi^{6}+\phi+3$, respectively.

Corollary 5.2. Let $n$ and $s$ be integers. Then,

$$
\begin{gathered}
\sum_{k=0}^{n} \sum_{p=0}^{k}\left(\begin{array}{l}
n \\
k
\end{array}\right)\left(\begin{array}{l}
k \\
p
\end{array}\right) \frac{G_{k+4 p+s}}{2^{k}}=2^{n} G_{3 n+s} \\
\sum_{k=1}^{n} \sum_{p=0}^{k}\left(\begin{array}{l}
n \\
k
\end{array}\right)\left(\begin{array}{l}
k \\
p
\end{array}\right) \frac{k G_{k+4 p+s}}{2^{k}}=2^{n-2} n\left(G_{3 n+s+2}+G_{3 n+s-2}\right)
\end{gathered}
$$

and

$$
\begin{gathered}
\sum_{k=0}^{n} \sum_{p=0}^{k}\left(\begin{array}{l}
n \\
k
\end{array}\right)\left(\begin{array}{l}
k \\
p
\end{array}\right) \frac{G_{k+5 p+s}}{3^{k}}=\left(\frac{7}{3}\right)^{n} G_{3 n+s}, \\
7 \sum_{k=1}^{n} \sum_{p=0}^{k}\left(\begin{array}{l}
n \\
k
\end{array}\right)\left(\begin{array}{l}
k \\
p
\end{array}\right) \frac{k G_{k+5 p+s}}{3^{k}}=\left(\frac{7}{3}\right)^{n} n\left(G_{3 n+s+3}+G_{3 n+s-2}\right) .
\end{gathered}
$$

Proof. Set $j=0$ and $j=1$ in (28) and (29), respectively.

\section{A general binomial sum identity}

Theorem 6.1. Let $j, s$ and $v$ be integers with $j, v \geq 0, v \neq 0, v \neq 1$. Then,

$$
\begin{aligned}
& \left(\begin{array}{c}
n \\
j
\end{array}\right) \sum_{m=0}^{j} \sum_{q=0}^{j-m}(-1)^{j+m+q}\left(\begin{array}{c}
j \\
j-m
\end{array}\right)\left(\begin{array}{c}
j-m \\
q
\end{array}\right)\left(\frac{T_{v}}{T_{v-1}}\right)^{m} G_{v n-j(v-1)+q+s} \\
& =\frac{T_{v-2}^{n}}{T_{v-1}^{j}} \sum_{k=j}^{n} \sum_{p=0}^{k} \sum_{w=0}^{k-p}(-1)^{k+w+p}\left(\begin{array}{c}
k \\
j
\end{array}\right)\left(\begin{array}{c}
n \\
k
\end{array}\right)\left(\begin{array}{c}
k \\
k-p
\end{array}\right)\left(\begin{array}{c}
k-p \\
w
\end{array}\right)\left(\frac{T_{v-1}}{T_{v-2}}\right)^{k}\left(\frac{T_{v}}{T_{v-1}}\right)^{p} G_{k+w+s} .
\end{aligned}
$$

Proof. For $v \geq 1$ and $\phi=\alpha$ write (4) in the form

$$
\alpha^{v}=\alpha\left(T_{v}+T_{v-1}(\alpha-1)\right)+T_{v-2} .
$$

Now, identify $x=\alpha\left(T_{v}+T_{v-1}(\alpha-1)\right)$ and $a=T_{v-2}$ and use Lemma 3.1 and the binomial theorem to get

$$
\begin{aligned}
\sum_{k=j}^{n}\left(\begin{array}{l}
k \\
j
\end{array}\right) & \left(\begin{array}{l}
n \\
k
\end{array}\right)(-1)^{k} T_{v-2}^{n-k} \sum_{p=0}^{k}\left(\begin{array}{l}
k \\
p
\end{array}\right) T_{v}^{p} T_{v-1}^{k-p} \sum_{w=0}^{k-p}\left(\begin{array}{c}
k-p \\
w
\end{array}\right)(-1)^{w+p} \alpha^{k+w} \\
& =\left(\begin{array}{c}
n \\
j
\end{array}\right) \sum_{m=0}^{j}\left(\begin{array}{c}
j \\
m
\end{array}\right) T_{v}^{m} T_{v-1}^{j-m} \sum_{q=0}^{j-m}\left(\begin{array}{c}
j-m \\
q
\end{array}\right)(-1)^{j-(m+q)} \alpha^{v n-j(v-1)+q} .
\end{aligned}
$$

Multiply both sides by $\alpha^{s}$ and combine the similar results for $\beta$ and $\gamma$ according to the Binet formula (1).

Corollary 6.1. We have

$$
\sum_{k=0}^{n} \sum_{p=0}^{k} \sum_{w=0}^{k-p}(-1)^{k+w+p}\left(\begin{array}{l}
n \\
k
\end{array}\right)\left(\begin{array}{c}
k \\
p
\end{array}\right)\left(\begin{array}{c}
k-p \\
w
\end{array}\right)\left(\frac{T_{v-1}}{T_{v-2}}\right)^{k}\left(\frac{T_{v}}{T_{v-1}}\right)^{p} G_{k+w+s}=\frac{G_{v n+s}}{T_{v-2}^{n}} .
$$

For $v=1$ the left-hand side collapses and we end with $G_{n+s}$ on both sides of the equality sign. The special values for $v=2$ and $v=3$ are given by

$$
\sum_{p=0}^{n} \sum_{w=0}^{n-p}(-1)^{w+p}\left(\begin{array}{c}
n \\
p
\end{array}\right)\left(\begin{array}{c}
n-p \\
w
\end{array}\right) G_{n+w+s}=(-1)^{n} G_{2 n+s}
$$

and

$$
\sum_{k=0}^{n} \sum_{p=0}^{k} \sum_{w=0}^{k-p}(-1)^{w+p+k}\left(\begin{array}{l}
n \\
k
\end{array}\right)\left(\begin{array}{c}
k \\
k-p
\end{array}\right)\left(\begin{array}{c}
k-p \\
w
\end{array}\right) 2^{p} G_{k+w+s}=G_{3 n+s}
$$




\section{References}

[1] K. Adegoke, Weighted tribonacci sums, Konuralp J. Math. 8 (2020) 355-360.

[2] K. Adegoke, R. Frontczak, T. Goy, Special sums with squared Horadam numbers and generalized Tribonacci numbers, Palest. J. Math. (2021), To appear.

[3] K. Adegoke, A. Olatinwo, W. Oyekanmi, New Tribonacci recurrence relations and addition formulas, Notes Number Theory Discrete Math. 26 (2020) 164-172.

[4] P. Anantakitpaisal, K. Kuhapatanakul, Reciprocal sums of the tribonacci numbers, J. Integer Seq. 19 (2016) \#16.2.1.

[5] K. N. Boyadzhiev, Notes on the Binomial Transform: Theory and Table with Appendix on Stirling Transform, World Scientific, Singapore, 2018.

[6] M. Catalani, Identities for Tribonacci-related sequences, arXiv:0209179 [math.CO], (2002).

[7] G. Cerda-Morales, Quadratic approximation of generalized tribonacci sequences, Discuss. Math. Gen. Algebra Appl. 38 (2018) $227-237$.

[8] E. Choi, Modular tribonacci numbers by matrix method, J. Korean Soc. Math. Educ. Ser. B Pure Appl. Math. 20 (2013) $207-221$.

[9] L. Comtet, Advanced Combinatorics: The Art of Finite and Infinite Expansions, D. Reidel, Dordrecht, 1974.

[10] J. Feng, More identities on the Tribonacci numbers, Ars Combin. 100 (2011) 73-78.

[11] R. Frontczak, A short remark on Horadam identities with binomial coefficients, Ann. Math. Inf. 54 (2021) DOI: 10.33039/ami.2021.03.016, In press.

[12] R. Frontczak, Convolutions for generalized Tribonacci numbers and related results, Int. J. Math. Anal. 12 (2018) 307-324.

[13] R. Frontczak, Relations for generalized Fibonacci and Tribonacci sequences, Notes Number Theory Discrete Math. 25 (2019) $178-192$.

[14] R. Frontczak, Sums of Tribonacci and Tribonacci-Lucas numbers, Int. J. Math. Anal. 12 (2018) 19-24.

[15] T. Goy, M. Shattuck, Determinant identities for Toeplitz-Hessenberg martices with tribonacci number entries, Trans. Comb. 9 (2020) 89-109.

[16] M. Janjić, Words and linear recurrences, J. Integer Seq. 21 (2018) \#18.1.4.

[17] T. Komatsu, R. Li, Convolution identities for Tribonacci numbers with symmetric formulae, Math. Rep. 21(71) (2019) $27-47$.

[18] K. Kuhapatanakul, L. Sukruan, The generalized tribonacci numbers with negative subscripts, Integers 14 (2014) \#A32.

[19] N. J. A. Sloane, The On-Line Encyclopedia of Integer Sequences, https ://oeis . org.

[20] Y. Soykan, Tribonacci and Tribonacci-Lucas matrix sequences with negative subscripts, Comm. Math. Appl. 11 (2020) 141-159.

[21] N. Yilmaz, N. Taskara, Tribonacci and Tribonacci-Lucas numbers via the determinants of special matrices, Appl. Math. Sci. 8 (2014) $1947-1955$. 